\title{
AVALIAÇÃO DA ACURÁCIA NO DIRECIONAMENTO COM PILOTO AUTOMÁTICO E CONTRASTE DA CAPACIDADE DE CAMPO OPERACIONAL NO PLANTIO MECANIZADO DA CANA-DE-AÇÚCAR
}

\section{FÁBIO H. R. BAIO ${ }^{1}$, RODRIGO F. MORATELLI ${ }^{2}$}

\begin{abstract}
RESUMO: Com o crescimento da mecanização da cana-de-açúcar, é necessária uma dinamização dos processos, como no uso de técnicas de agricultura de precisão para uma diminuição do custo de produção. O presente trabalho foi desenvolvido com o objetivo de contrastar a acurácia nas passadas orientadas por dois sistemas de direcionamento, autodirecionamento via satélite versus manual, assim como comparar as capacidades de campo e a eficiência operacional envolvidos no plantio mecanizado da cana-de-açúcar. Foram analisados dois tratamentos: com e sem o auxílio do piloto automático por GPS RTK na operação de plantio mecanizado. As avaliações foram realizadas em condições de campo, na Usina IACO Agrícola S.A., em Chapadão do Sul - MS. De acordo com os resultados obtidos, afirmou-se que o uso do piloto automático na operação de plantio da cana-de-açúcar ofereceu uma acurácia de 0,033 m entre as passadas, sendo cinco vezes maior do que aquela obtida com o direcionamento manual, e que o seu uso não melhorou a eficiência operacional do conjunto mecanizado na operação de plantio, sendo limitada pelo sistema de colheita e transporte da cana muda para a plantadora.
\end{abstract}

PALAVRAS-CHAVE: piloto automático, direcionamento, GPS.

\section{AUTO GUIDANCE ACCURACY EVALUATION AND CONTRAST OF THE OPERATIONAL FIELD CAPACITY ON THE MECHANIZED PLANTATION SYSTEM OF SUGAR CANE}

\begin{abstract}
With the growth of mechanization on the sugar cane plantation, the processes need to be speeded up, such as with the use of precision farming techniques to decrease the production cost. The purpose of this study was to compare the accuracy achieved by an auto guidance system driving the passes of a sugar cane planter machine over the field versus the manual system, compare the operational field capacity and compare the operational efficiency. Two treatments were analyzed: auto guidance by RTK GPS and manual guidance on the sugar cane planting operation. The evaluations were conducted under field conditions at IACO Agrícola S/A mill, in Chapadão do Sul-MS. According to the results, it was stated that the use of auto guidance system on sugar cane planting operation gathered an accuracy of $0.033 \mathrm{~m}$ pass to pass. This accuracy is five times greater than the obtained by the manual steering system. The use of an auto guidance steering did not increase the operational efficiency of the mechanized system, which is limited by the harvesting and transporting system of the sugar cane seeding to the planter.
\end{abstract}

KEYWORDS: auto pilot, guidance, GPS.

\section{INTRODUÇÃO}

Recentemente, técnicas de agricultura de precisão estão sendo aplicadas para a diminuição do custo de produção de etanol e pelo menor uso de insumos agrícolas, contribuindo para melhorar o balanço energético na produção de etanol a partir da cana-de-açúcar. O uso de sistemas de orientação por satélites pode auxiliar na otimização do parque de máquinas pela redução de conjuntos mecanizados para uma determinada operação. De acordo com STABILEI \& BALASTREIRE (2006), o alto custo de aquisição do GPS tem detido um pleno avanço da

\footnotetext{
${ }^{1}$ Eng ${ }^{\circ}$ Agrônomo, Prof. Adjunto, CPCS/UFMS, Chapadão do Sul - MS, Fone (OXX67) 3562.6300, fabiobaio@ufms.br.

${ }^{2}$ CPCS/UFMS, Chapadão do Sul - MS, rodrigo.moratelli@gmail.com.

Recebido pelo Conselho Editorial em: 6-10-2009

Aprovado pelo Conselho Editorial em: 25-2-2011
} 
Agricultura de Precisão no Brasil. Esta afirmação é uma verdade até os dias atuais, uma vez que grande parte destes equipamentos é importada e com elevada tributação alfandegária. Dentre vários modelos de receptores GPS, podem-se citar modelos de GPS com correção diferencial do erro do sistema GPS por RTK (Real Time Kinematic), que atualmente são os que fornecem a maior acurácia no posicionamento em tempo real (TRIMBLE, 2010). O grande benefício dos pilotos automáticos é a redução de erros entre as passadas pela substituição do operador no direcionamento durante as operações mecanizadas. SILVA et al. (2011) pesquisaram que 39\% das usinas de cana-de-açúcar instaladas no Brasil adotam sistemas de autodirecionamento por satélites, também popularizados por pilotos automáticos.

BALASTREIRE \& BAIO (2002) mostraram que o posicionamento relativo com DGPS (Differential Global Positioning System) pode ser substituído por algum sistema que utiliza um algoritmo para a melhoria da acurácia no posicionamento, como o algoritmo e-Dif ${ }^{\circledR}$ (HEMISPHERE, 2010), que corrige o posicionamento horizontal sem nenhum tipo de sinal diferencial, alcançando coordenadas tão precisas quanto as obtidas pela utilização do DGPS. No entanto, estes algoritmos não permitem posicionamento acurado e, sim, preciso. De acordo com BAIO (2007), algumas aplicações agrícolas exigem acurácia tão elevada que os sistemas DGPS não podem oferecer, como na sulcação ou no plantio mecanizado da cana-de-açúcar, exigindo-se a correção RTK nestas aplicações. O autor ainda relata que o setor canavieiro está investindo cada vez mais na tecnologia de sistemas de direcionamento via satélite, que também está sendo chamado de autodirecionamento, ou piloto automático. SHOCKLEY \& DILLON (2008) relatam que o piloto automático reduz sobreposições e falhas na aplicação, pode elevar a velocidade operacional, possibilita maior acurácia na aplicação de insumos e eleva o tempo disponível para a operação. BATTE \& EHSANI (2006) mostram que a redução dos custos operacionais pela utilização desta tecnologia pode ser substancial.

O presente trabalho foi desenvolvido com o objetivo de contrastar a acurácia nas passadas orientadas por dois sistemas de direcionamento, autodirecionamento via satélite versus manual, assim como comparar as capacidades de campo e a eficiência operacional envolvidas no plantio mecanizado da cana-de-açúcar.

\section{MATERIAL E MÉTODOS}

As avaliações foram realizadas na fazenda IACO Agrícola S/A, no município de Chapadão do Sul - MS, com coordenadas aproximadas de 1846'20'S e 5256'40'WG. Houve uma concentração da coleta de dados em campo dentro do mês de novembro de 2009, não sendo observadas atividades solares expressivas dentro do período em avaliação, que poderiam afetar o resultado comparativo. $\mathrm{O}$ talhão utilizado para a coleta de dados, com 173,720 ha e declividade menor que $2 \%$, possui um formato retangular, comum na maioria das usinas de cana, e está posicionado de forma adequada para a recepção do sinal de correção da base RTK, sem interrupção física para o trânsito do sinal de rádio entre a base RTK e o equipamento em campo.

O trator utilizado foi um John Deere modelo 7815, com $148 \mathrm{~kW}$, ano de fabricação 2007, e com 250 horas de trabalho quando do início da coleta de dados. Neste trator, estava instalado um sistema de autodirecionamento da Trimble (Westminster, CO, EUA), modelo AutoPilot, com um receptor GPS AgGPS 262 e um controlador modelo AgGPS NavController II, com correção por base RTK, também fabricado pela Trimble, modelo AgGPS RTK 450 e com transmissão do sinal de correção RTK por rádio de modelo HPB450. O tempo de adiantamento do receptor GPS foi configurado em $1 \mathrm{~s}$, assim como o intervalo de registro dos dados. A base RTK estava posicionada em um ponto georreferenciado a $2 \mathrm{~km}$ do talhão, e a idade máxima do sinal da base foi configurada em $30 \mathrm{~s}$.

O implemento utilizado nas avaliações foi uma plantadora de cana-de-açúcar picada fabricada pela DMB (Sertãozinho - SP) modelo PCP 6000, ano 2008. Esta realizou a operação de plantio mecanizado de cana picada em um espaçamento de 1,4 $\mathrm{m}$ entre fileiras de plantio, dispostas de 
forma retilínea no talhão. A velocidade de plantio sempre foi mantida constante em ambos os tratamentos a $1,6 \mathrm{~m} \mathrm{~s}^{-1}\left(5,6 \mathrm{~km} \mathrm{~h}^{-1}\right)$.

Para a comparação entre a eficiência operacional e a capacidade de campo operacional entre os dois sistemas, foi utilizada a metodologia descrita por MIALHE (1974) e por BALASTREIRE (2007). Os dados coletados consistiram no tempo de execução das manobras, paradas para abastecimento de mudas de cana, paradas para abastecimento de adubo, paradas por embuchamento e tempo para completar a linha de plantio, cronometrados por um cronômetro digital portátil Cronobio, modelo SW2018. Durante todo o tempo em campo, houve a gravação da localização do trator por suas coordenadas calculadas pelo GPS, em ambos os tratamentos. As coordenadas dos pontos gerados pelas passadas em campo foram exportadas para um programa de SIG (Sistema de Informações Geográficas) SSToolbox, versão 3.8.0 (SST, 2010), onde estes dados foram manipulados, e os mapas das passadas foram gerados. Pelo SIG, as coordenadas dos pontos foram exportadas para uma planilha eletrônica onde os cálculos dos desvios puderam ser calculados. Estas coordenadas geográficas foram convertidas para a Projeção Transversa de Mercator (UTM) e rotacionadas.

Os cálculos para o dimensionamento dos erros em cada amostra foram realizados por uma planilha eletrônica desenvolvida por POVH (2007). Segundo o autor, se os sistemas de orientação fossem perfeitos, as coordenadas de cada passada do trator seriam exatamente iguais às linhas de referência, portanto o cálculo dos erros baseia-se simplesmente na diferença no eixo das abscissas entre a coordenada de cada ponto coletado em campo com sua respectiva referência. Os dados de saída da planilha separam os resultados automaticamente, de maneira que o conjunto de dados de cada passada fique em uma coluna diferente, com os erros em metros. A planilha considerou erros positivos e negativos, para a direita e esquerda da passada planejada, respectivamente.

O experimento foi constituído por dois tratamentos em blocos ao acaso: autodirecionamento via satélite, ou piloto automático, e direcionamento manual. Em um mesmo talhão agrícola, com o mesmo conjunto mecanizado e com o mesmo operador, foram realizados os tratamentos citados em dias diferentes, onde cada bloco foi caracterizado por uma passada do trator, e as mensurações dos erros em cada passada, por repetições. Foram calculados os intervalos de confiança entre os erros dos tratamentos a 95\% de probabilidade, assim como suas médias comparadas pelo teste de Tukey, com auxílio do programa SISVAR, versão 5.1 (FERREIRA, 2008). Também foi realizada uma análise não paramétrica dos resultados obtidos em campo, como sugere BAIO (2005).

Esta análise é realizada pela comparação gráfica dos intervalos de confiança, possibilitando uma visualização da provável variação do erro em torno da média, em um determinado nível de probabilidade. Também foi elaborado um gráfico ilustrando a frequência relativa acumulada dos desvios em relação ao alinhamento teórico. Este gráfico ilustra qual a porcentagem de amostras que se enquadra acima de um limite de erro aceitável.

\section{RESULTADOS E DISCUSSÃO}

$\mathrm{Na}$ operação do plantio, foram levantados 3.808 pontos amostrais, sendo que 1.837 destes foram gravados com o piloto automático ativado e $1.971 \mathrm{com}$ o direcionamento manual (piloto automático desativado e tela do computador de bordo coberta). Estes pontos foram tomados aleatoriamente das passadas gravadas em campo, em ambos os tratamentos. Observa-se pela Figura 1 que as passadas da plantadora de cana seguiram um direcionamento SE para NO, e vice-versa. A orientação das passadas é um relato importante e deve ser reportado, pois também afeta a acurácia do sistema, como mostra STOMBAUGH et al. (2008). 


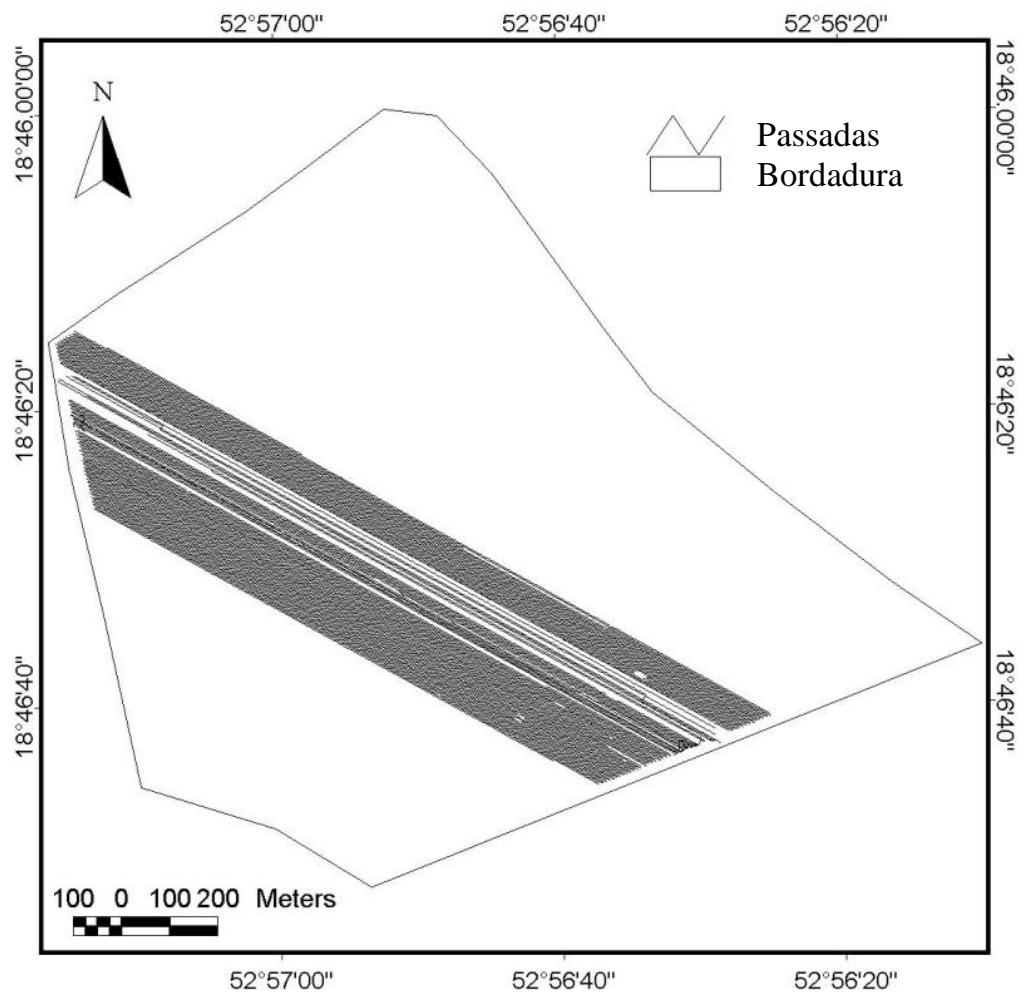

FIGURA 1. Área utilizada durante o ensaio e orientação das passadas em campo. Illustration of the field and orientation of the passes during the test.

Os resultados de uma análise estatística descritiva estão apresentados nas Tabelas 1 e 2 . Observa-se, na Tabela 1, pelos dados obtidos com o piloto automático ativado, que o erro médio foi de $0,033 \mathrm{~m}$, enquanto na Tabela 2 , pelos dados obtidos com o direcionamento manual, onde o operador tomou por base uma passada referencial anterior, o erro médio foi de 0,170 $\mathrm{m}$.

De acordo com um fabricante de sistema de piloto automático (JOHN DEERE, 2009), a acurácia deste sistema trabalhando com a correção RTK é de $0,0025 \mathrm{~m}$, em $95 \%$ do tempo. Entretanto, essa acurácia é mensurada em condições estáticas.

TABELA 1. Erros médios (m), mínimos e máximos em cada passada (L0 a L7), desvio-padrão e coeficiente de variação (C.V.) obtidos com o piloto automático ativado, na operação de plantio da cana-de-açúcar. Average, minimum and maximum errors (m) of each pass (LO to L7), standard deviation and variation coefficient (CV) obtained by auto guidance on the mechanized plantation system of sugar cane.

\begin{tabular}{lrrrrrrrrr}
\hline & L0 & \multicolumn{1}{c}{ L1 } & \multicolumn{1}{c}{ L2 } & L3 & \multicolumn{1}{c}{ L4 } & L5 & L6 & L7 & Média \\
\hline Média & 0,033 & 0,030 & 0,031 & 0,037 & 0,030 & 0,031 & 0,033 & 0,047 & 0,033 \\
Mínimo & $-0,250$ & $-0,159$ & $-0,104$ & $-0,120$ & $-0,116$ & $-0,137$ & $-0,103$ & $-0,110$ & $-0,250$ \\
Máximo & 0,085 & 0,087 & 0,087 & 0,100 & 0,117 & 0,102 & 0,098 & 0,017 & 0,117 \\
Desvio-Padrão & 0,028 & 0,025 & 0,023 & 0,025 & 0,024 & 0,023 & 0,023 & 0,027 & 0,025 \\
C.V. & 0,850 & 0,821 & 0,740 & 0,668 & 0,796 & 0,749 & 0,691 & 0,578 & 0,755 \\
Assimetria & 2,696 & 1,511 & 0,794 & 0,685 & 1,111 & 0,962 & 0,798 & 0,205 & 1,268 \\
Curtose & 15,616 & 3,759 & $-0,070$ & 0,130 & 1,146 & 1,161 & 0,074 & $-0,355$ & 3,869 \\
\hline
\end{tabular}

Também é notado pela Tabela 1 que o maior erro médio nas passadas, com o uso do piloto automático, foi negativo. O sinal negativo indicou que o erro foi à esquerda da linha planejada, ou seja, o piloto automático "fechou" a linha de plantio, aproximando-se da linha anterior e diminuindo o espaçamento planejado. Com o operador controlando o direcionamento da máquina, o maior erro chegou a atingir $0,706 \mathrm{~m}$ à esquerda (negativo), reduzindo praticamente pela metade o espaçamento 
desejado entre linhas de 1,4 $\mathrm{m}$. Ainda, este erro teve pouca influência pela declividade do terreno atuando sobre o escorregamento da máquina, já que foi menor do que $2 \%$.

Os coeficientes de variação obtidos em ambos os tratamentos foram semelhantes, induzindo à ideia de que a variabilidade dos dados em torno das médias foi semelhante também; contudo, os valores de curtose indicam que o tratamento com o piloto automático obteve um conjunto de amostras muito mais homogêneo, ou seja, a curva de distribuição é leptocúrtica quando comparada à distribuição normal.

TABELA 2. Erros médios (m), mínimos e máximos em cada passada, desvio-padrão e coeficiente de variação (C.V.) obtidos com o direcionamento manual, na operação de plantio da cana-de-açúcar. Average, minimum and maximum errors $(\mathrm{m})$ of each pass (LO to L7), standard deviation and variation coefficient (CV) obtained by the manual guidance on the mechanized plantation system of sugar cane.

\begin{tabular}{lrrrrrrrrr}
\hline & \multicolumn{1}{c}{ L0 } & \multicolumn{1}{c}{ L1 } & \multicolumn{1}{c}{ L2 } & \multicolumn{1}{c}{ L3 } & \multicolumn{1}{c}{ L4 } & \multicolumn{1}{c}{ L5 } & \multicolumn{1}{c}{ L6 } & \multicolumn{1}{c}{ L7 } & Média \\
\hline Média & 0,123 & 0,145 & 0,152 & 0,186 & 0,200 & 0,196 & 0,197 & 0,157 & 0,170 \\
Mínimo & $-0,441$ & $-0,706$ & $-0,562$ & $-0,504$ & $-0,471$ & $-0,275$ & $-0,242$ & $-0,268$ & $-0,706$ \\
Máximo & 0,341 & 0,535 & 0,520 & 0,572 & 0,573 & 0,590 & 0,525 & 0,457 & 0,341 \\
Desv.-Padrão & 0,104 & 0,124 & 0,108 & 0,136 & 0,146 & 0,126 & 0,134 & 0,114 & 0,127 \\
CV & 0,841 & 0,857 & 0,710 & 0,732 & 0,730 & 0,642 & 0,677 & 0,722 & 0,750 \\
Assimetria & 1,160 & 1,744 & 1,109 & 0,864 & 0,574 & 0,356 & 0,463 & 0,523 & 0,857 \\
Curtose & 0,794 & 3,860 & 1,491 & 0,223 & $-0,569$ & $-0,701$ & $-0,886$ & $-0,651$ & 0,227 \\
\hline
\end{tabular}

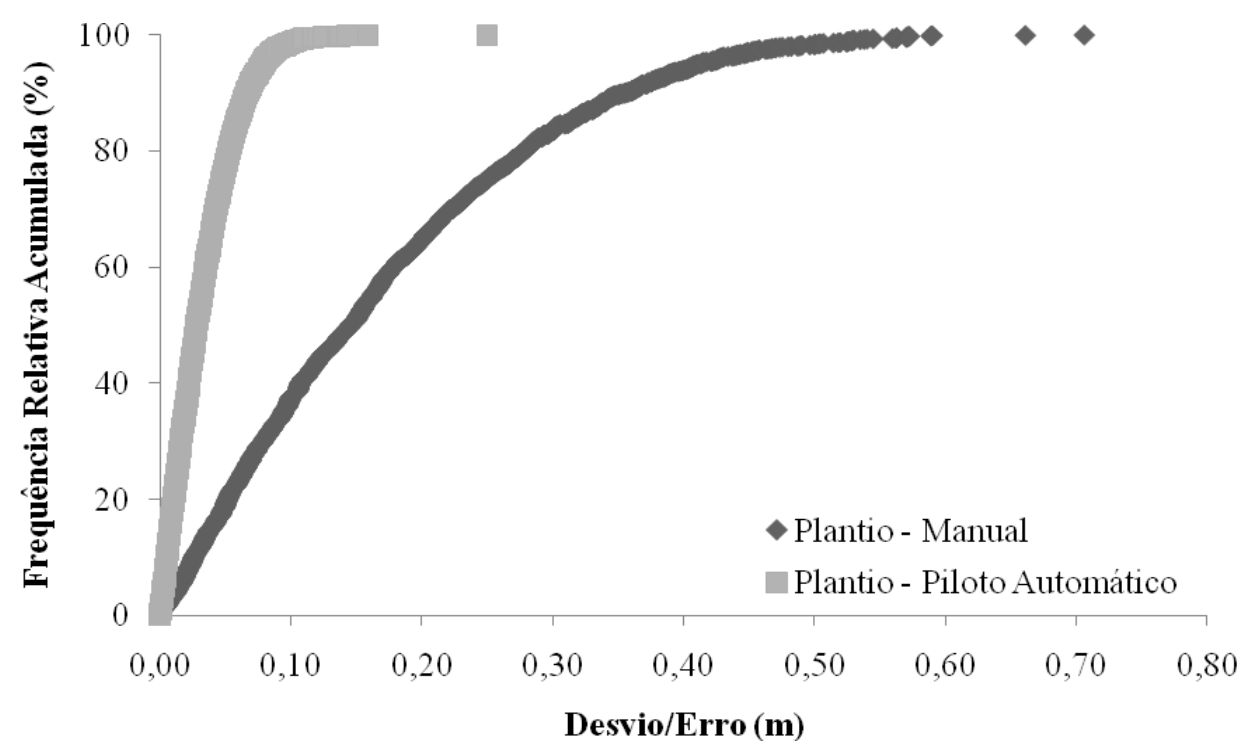

FIGURA 2. Frequência relativa acumulada dos desvios (\%) no ensaio com e sem o sistema de autodirecionamento por piloto automático. Cumulative frequency of the errors (\%) obtained on the treatments with auto guidance and manual system.

Na Figura 2, pode ser observada a frequência relativa acumulada dos desvios (\%) para cada tratamento no ensaio com o sistema de autodirecionamento por piloto automático. Verifica-se que 90\% das observações dos desvios obtidos com o sistema de piloto automático foram menores que $0,066 \mathrm{~m}$, sendo que o maior desvio foi de $0,249 \mathrm{~m}$. Já sem o sistema de piloto automático em operação, $90 \%$ das observações dos desvios foram menores que $0,356 \mathrm{~m}$, sendo o maior desvio de $0,706 \mathrm{~m}$.

Foi calculado o intervalo de confiança (IC), ao nível de 95\% de significância, para todas as observações na operação de plantio, como sugerido por BAIO (2005). O valor do IC encontrado 
para o piloto automático foi de $0,033 \pm 0,001 \mathrm{~m}$, conforme pode ser visualizado graficamente pela Figura 3; assim, neste nível de significância indicado e quando todas as condições operacionais e ambientais forem as mesmas das do ensaio, o erro provável estará entre 0,032 e $0,034 \mathrm{~m}$, com $95 \%$ de probabilidade. Na Figura 3, ilustra-se o gráfico comparativo dos intervalos de confiança para os diferentes tratamentos. Com o direcionamento manual, o valor do IC foi de 0,170 $\pm 0,006 \mathrm{~m}$, demonstrando que, com o nível de significância indicado e nas mesmas condições, o erro provável estará entre 0,164 e $0,176 \mathrm{~m}$, com $95 \%$ de probabilidade.

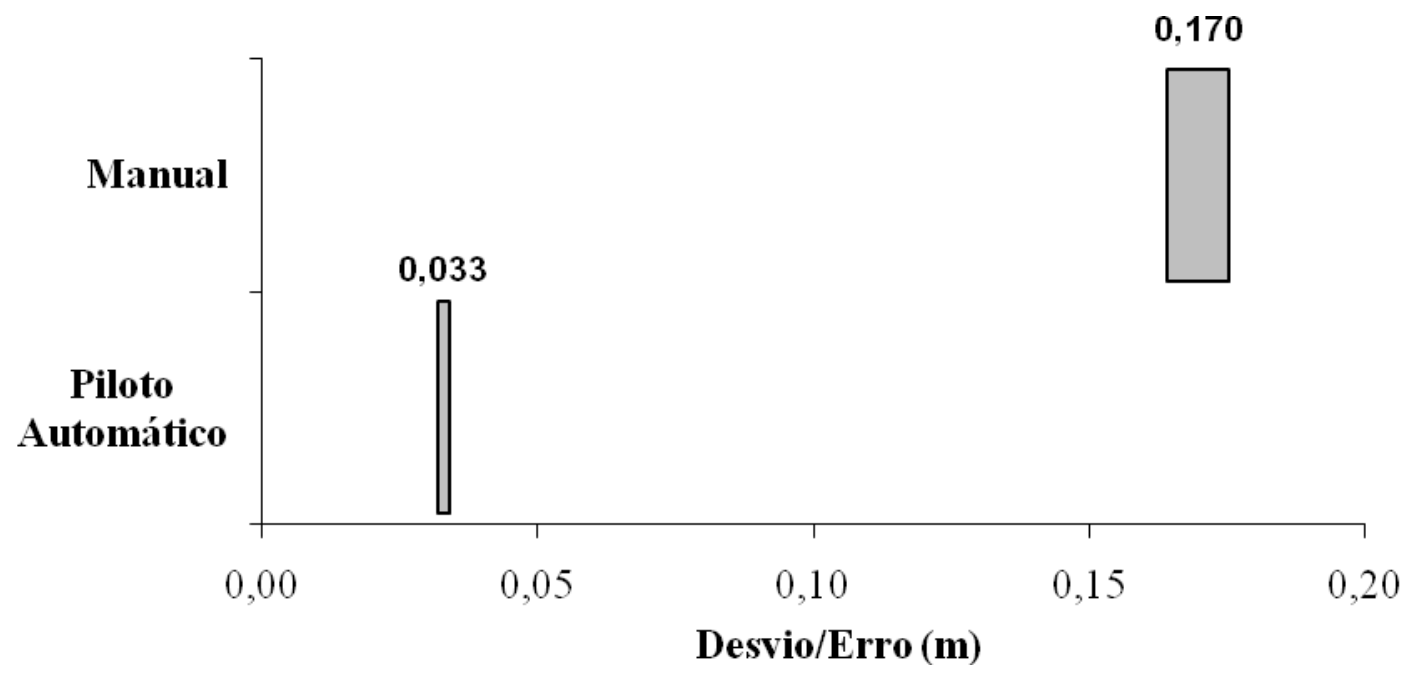

FIGURA 3. Comparativo dos intervalos de confiança (IC) e desvio médio (m) obtidos para cada tratamento na operação de plantio mecanizado da cana. Comparative of confidence interval and average error $(\mathrm{m})$ gathered on each treatment on the mechanized plantation system of sugar cane.

$\mathrm{Na}$ Tabela 3, está apresentado o teste de comparação das médias por Tukey, a 5\% de probabilidade, comprovando a diferença estatística entre os dois métodos de direcionamento, onde o direcionamento por piloto automático apresentou um erro cinco vezes inferior ao manual, aproximadamente.

TABELA 3. Teste de Tukey para a comparação entre as médias (m) dos tratamentos com piloto automático e manual, na operação de plantio de cana-de-açúcar (DMS - Diferença Mínima Significativa: 0,0058). Tukey's test comparison of the treatments with auto guidance and manual during the mechanized plantation of sugar cane (Significant Minimum Difference: 0,0058).

\begin{tabular}{ccc}
\hline Tratamento & Média $(\mathrm{m})$ & Tukey* \\
\hline Piloto Automático & 0,033 & $\mathrm{~B}$ \\
Manual & 0,170 & $\mathrm{~A}$ \\
\hline
\end{tabular}

* Médias seguidas de mesma letra não diferem entre si, pelo teste de Tukey, a 5\% de significância.

O espaçamento irregular entre sulcos é obtido na operação de sulcação ou plantio mecanizado e gera dificuldades para todas as operações subsequentes, afetando todo o processo produtivo. $\mathrm{Na}$ reforma dos canaviais, a irregularidade no espaçamento aparece como a causa dos efeitos na operação de eliminação mecânica da soqueira, gerando falhas no arranquio. Durante o plantio mecanizado, é gerada uma redução do estande, e nas operações de cultivo tríplice, colheita e aplicação de herbicidas, o alinhamento das máquinas é afetado. As máquinas e implementos trabalham com espaçamento fixo, e, se as fileiras estão desalinhadas e fora dos limites de tolerância especificados, as dificuldades nas operações são evidentes, como exemplo, o pisoteio das fileiras e as falhas na pulverização (CAMPOS et al., 2008). Considerando um talhão quadrático de $100 \mathrm{~m}$ por 
$100 \mathrm{~m}$, com as passadas sendo executadas com direcionamento perfeito e espaçamento entre passadas da plantadora de $2,800 \mathrm{~m}$, seriam possíveis 35,710 passadas com duas linhas de plantio, ou seja, 71,420 linhas de plantio no total. Com o uso do piloto automático, e considerando-se o erro médio de 0,033 $\mathrm{m}$ acumulado ao longo das passadas, o número total de passadas de plantio com duas linhas seria de 35,330 passadas, ou uma redução de apenas $1 \%$ sobre o total teórico. Já com o direcionamento manual e com o erro médio entre passadas de $0,170 \mathrm{~m}$, há uma redução no número total para 33,780 passadas possíveis, ou uma redução de 5\% na quantidade de linhas de plantio neste talhão ilustrativo. Ao se extrapolar esses números para valores de produção em toneladas de cana-de-açúcar, considerando-se o único fator teórico afetando a produtividade como sendo o espaçamento de plantio e estimando-se uma produtividade de $100 \mathrm{t} \mathrm{ha}^{-1}$, em um hectare de plantio no espaçamento de 1,400 $\mathrm{m}(7.142,860 \mathrm{~m})$, seriam produzidos em média $14 \mathrm{~kg} \cdot \mathrm{m}^{-1}$. Ao considerar que o plantio com o direcionamento manual resultaria em 2,860 linhas de plantio a menos quando comparado ao piloto automático, haveria uma redução na produtividade em $4.000 \mathrm{~kg} \mathrm{ha}^{-1}$.

Para o cálculo da Capacidade de Campo Operacional, todos os tempos foram tomados em campo (tempo de manobra, abastecimento da plantadora com as mudas, abastecimento da plantadora com adubo, embuchamento da esteira dosadora ou do sulcador, desvios necessários devido a carreadores e linhas de energia que passam pelo talhão e outras paradas eventuais). $\mathrm{O}$ cálculo da eficiência operacional foi baseado no tempo em que a máquina esteve em trabalho efetivo (tempo total em campo menos o tempo total de perda), dividido pelo tempo total. As eficiências da operação de plantio estão na Tabela 4. O cálculo da eficiência operacional apresentou resultados semelhantes, próximos a 70\%. De acordo com ASAE (2000), a eficiência de campo de uma plantadora deve estar entre $50 \%$ e $75 \%$. Assim, ambos os tratamentos apresentaram resultados semelhantes e adequados de eficiência. Como a velocidade e a largura efetiva da plantadora de cana foram as mesmas em ambos os tratamentos, a variação da capacidade de campo operacional foi exclusivamente influenciada pela eficiência operacional, não diferindo estatisticamente em ambos os tratamentos. $\mathrm{O}$ fator que mais contribuiu para a redução na eficiência operacional foi o tempo de espera por cana muda, totalizando $40 \%$ do tempo perdido, seguido pelo tempo de manobra (19\%) e pelo tempo para abastecimento de adubo (11\%); todavia, não houve diferença pelo uso do sistema com o piloto automático. Assim, a capacidade de campo operacional da plantadora de cana foi limitada pelo sistema de colheita e transporte da cana muda (fator limitante), dentro do sistema mecanizado de plantio como um todo.

TABELA 4. Eficiência operacional e capacidade de campo operacional de uma plantadora de cana-de-açúcar, com direcionamento por piloto automático e manual. Operational efficiency and operational field capacity comparison of a sugar cane planter operating with an auto guidance system versus manual system.

\begin{tabular}{lcc}
\hline Tratamento & $\begin{array}{c}\text { Eficiência } \\
\text { Operacional }\end{array}$ & $\begin{array}{c}\text { Capacidade de Campo } \\
\text { Operacional }\left(\mathrm{ha} \mathrm{h}^{-1}\right)\end{array}$ \\
\hline Piloto Automático & $69 \%$ & 1,430 \\
Manual & $68 \%$ & 1,410 \\
\hline
\end{tabular}

\section{CONCLUSÕES}

O uso do piloto automático na operação de plantio da cana-de-açúcar ofereceu uma acurácia de $0,033 \mathrm{~m}$ entre as passadas, sendo cinco vezes maior do que aquela obtida com o direcionamento manual e possibilitando o plantio de maior quantidade de linhas por área.

O uso do piloto automático não melhorou a eficiência operacional do conjunto mecanizado na operação de plantio. A capacidade de campo operacional da plantadora de cana foi igual em ambos os tratamentos, e foi limitada pelo sistema de colheita e transporte da cana muda. 


\section{REFERÊNCIAS}

ASAE. AMERICAN SOCIETY OF AGRICULTURAL ENGINEERS. ASAE D497.4: agricultural machinery management data. $47^{\text {th }}$ ed. St. Joseph: ASAE, 2000. p.350-357.

BAIO, F.H.R. Aplicação de AP no Plantio. In: RIPOLI, T.C.C.; RIPOLI, M.L.C.; CASAGRANDI, D.V.; IDE, B.Y. (Org.). Plantio de cana-de-açúcar: estado da arte. 2.ed. Piracicaba: T.C.C.Ripoli, 2007, v.1, p.92-101.

BAIO, F.H.R. Metodologia para ensaio de sistemas de direcionamento via satélite em percursos retos e curvos. 2005. 100 f. Tese (Doutorado em Energia na Agricultura) - Faculdade de Ciências Agronômicas, Universidade Estadual Paulista, Botucatu, 2005

BALASTREIRE, L.A. Máquinas agrícolas. 3.ed. Piracicaba, 2007. 307 p.

BALASTREIRE, L.A.; BAIO, F.H.R. Avaliação do desempenho de um GPS com algoritmo otimizado sem sinal de correção para a agricultura de precisão. In: BALASTREIRE, L.A. Avanços na agricultura de precisão no Brasil no período de 1998-2001. Piracicaba: L.A. Balastreire, 2002. p.285-288.

BATTE, M.T.; EHSANI, M.R. The economics of precision guidance wiht auto-boom control for farmer-owned agricultural sprayers. Computers and Eletronics in Agriculture, Amsterdam, v.53, p.28-44, 2006.

CAMPOS, C.M.; MILAN, M.; SIQUEIRA, L.F.F. Identificação e avaliação de variáveis críticas no processo de produção da cana-de-açúcar. Engenharia Agrícola, Jaboticabal, v.28, n.3, 2008.

Disponível em <http://www.scielo.br/scielo.php?script=sci_arttext\&pid=S0100-

69162008000300016\&lng=pt\&nrm=iso>. Acesso em: 29 jul. 2009.

FERREIRA, D.F. Sisvar: um programa para análises e ensino de estatística. Symposium, Campinas, v.6, p.36-41, 2008.

HEMISPHERE. e-Dif. Disponível em: <http://www.canalgeomatics.com/product_details.php? product_id=149>. Acesso em: 5 out. 2010.

JOHN DEERE. AMS - Soluções em Gerenciamento Agrícola. Disponível em:

<http://www.deere.com.br/pt_BR/ag/products/ams/base_rtk.html>. Acesso em: 30 jul. 2009.

MIALHE, L.G. Manual de mecanização agrícola. São Paulo: Agronômica Ceres, 1974. 301 p.

POVH, F.P.; SPEKKEN, M.; SALVI, J.V.; MACHADO, T.M.; MOLIN, J.P. Metodologia para análise de paralelismo em sistemas de orientação utilizando planilha eletrônica. In: SIMPÓSIO INTERNACIONAL DE AGRICULTURA DE PRECISÃO, 4., 2007, Viçosa. Anais... Viçosa: UFV, 2007. 1 CD-ROM.

SHOCKLEY, J.M.; DILLON, C.R. Cost savings for multiple inputs with swath control and auto guidance technologies. In: INTERNATIONAL CONFERENCE ON PRECISION AGRICULTURE, 9., 2008, Denver. Proceedings... Denver: Colorado State University, 2008. 1 CDROM.

SILVA, C.B.; MORAES, M.A.F.D.; MOLIN, J.P. Adoption and use of precision agriculture technologies in the sugarcane industry of São Paulo state, Brazil. Precision Agriculture, v.12, n.1., p.67-81, 2011.

SST SSToolbox - SST Development Group. Disponível em: <http://www.sstsoftware.com/ sstoolbox.htm>. Acesso em: 5 out. 2010.

STABILE, M.C.C.; BALASTREIRE, L.A. Comparação de três receptores GPS para uso em agricultura de precisão. Engenharia Agrícola, Jaboticabal, v.26, n.1, p.215-223, 2006.

STOMBAUGH, T.S.; SAMA, M.P.; ZANDONADI, R.S.; SHEARER, S.A.; KOOSTRA, B.K. Implications of standardized GNSS accuracy testing. In: INTERNATIONAL CONFERENCE ON 
PRECISION AGRICULTURE, 9., 2008, Denver. Proceedings... Denver: Colorado State University, 2008. 1 CD-ROM.

TRIMBLE, G. 2010. Disponível em: < http://www.trimble.com/agriculture/ guidance.aspx/> Acesso em: 20 dez. 2010. 